\title{
Respiratory Support in Severely or Critically Ill ICU Patients With COVID-19 in Wuhan, China
}

\author{
Hong-tao $\mathrm{HU}^{1 \dagger}$, Shen $\mathrm{XU}^{2 \dagger}$, Jing WANG ${ }^{1}$, Xin $\mathrm{RAO}^{\#}$ \\ ${ }^{1}$ Department of Critical Care Medicine, Zhongnan Hospital of Wuhan University, Wuhan 430071, China \\ ${ }^{2}$ Department of Nephrology, General Hospital of the Yangtze River Shipping, Wuhan 430010, China
}

(C) Huazhong University of Science and Technology 2020

\begin{abstract}
Summary: This case series aimed to describe the clinical characteristics of severely or critically ill patients with COVID-19 and compare the clinical characteristics of patients who received invasive respiratory support with those of patients who received noninvasive respiratory support. We included all confirmed severe or critical illness cases of COVID-19 admitted to the Intensive Care Unit (ICU) of Zhongnan Hospital of Wuhan University, a COVID-19-designated hospital, from January 8 to March 12, 2020. Cases were analyzed for epidemiological, demographic, clinical, APACHE II, SOFA, radiological features and laboratory data. Outcomes of all patients were followed up as of March 12, 2020. This newly emerging virus had caused 55 confirmed severe or critical illness cases in ICU of a COVID-19-designated hospital. Most of the infected patients were men; more than half had underlying diseases, including hypertension, coronary artery disease and diabetes. The median age was 63 years old. Common symptoms at onset of illness were fever, fatigue and dry cough. Five $(9.1 \%)$ hospitalized patients were presumed to have been infected in the hospital, and $4(7.3 \%)$ health care workers were infected in their work. Of the 55 confirmed severe or critical illness cases, $10(18.2 \%)$ patients died during the follow-up period as of March 12 with the median follow-up period of 28 days (interquartile range 16-35). Nine patients received VV-ECMO for severe respiratory failure and $4(44.4 \%)$ patients died. Moreover, 28 patients received invasive respiratory support and $14(50.0 \%)$ patients died. In this single-center study, 55 severely or critically ill ICU patients were confirmed to have COVID-19 in Wuhan and the overall mortality was $29.1 \%$. Totally $28(50.9 \%)$ of severely or critically ill ICU patients received invasive respiratory support and $14(50.0 \%)$ died during the follow-up period.
\end{abstract}

Key words: COVID-19; SARS-CoV-2; acute respiratory distress syndrome; intensive care unit; respiratory support

In December 2019, a series of pneumonia cases of unknown cause were reported from Wuhan, Hubei Province, China ${ }^{[1-3]}$. On February 11, 2020, the World Health Organization (WHO) introduced the agency's official name of this disease as coronavirus disease 2019 (COVID-19) instead of novel coronavirusinfected pneumonia (NCIP) and decided to call the virus severe acute respiratory syndrome coronavirus 2 (SARS-CoV-2).

The disease has rapidly spread from Wuhan to other areas, and case reports have confirmed humanto-human transmission among COVID-19 patients $^{[3,4]}$. As of May 1, 2020, over 80 thousand COVID-19 cases in China had been confirmed, and more than 3 thousand patients had died. Among these cases, more than 3000

Hong-tao HU, E-mail: huht2014@126.com; Shen XU, E-mail: snk_xs2016@163.com

${ }^{\dagger}$ The authors contributed equally to this work.

${ }^{*}$ Corresponding author, E-mail: raoxin4169@163.com cases of COVID-19 in medical staff had been reported in China, 1716 had been confirmed, and 6 medical staff had died ${ }^{[5]}$.

In the early stage of COVID-19, severe acute respiratory infection symptoms occur, with some patients rapidly developing acute respiratory distress syndrome (ARDS), acute respiratory failure, and other serious complications. Wang et al described the clinical characteristics of 138 hospitalized patients with COVID-19 and indicated that most of the patients need respiratory support ${ }^{66}$. However, the difference in clinical characteristics between patients receiving invasive respiratory support (IRS) and those receiving noninvasive respiratory support (NIRS) has not been reported. The objective of this case series was to describe the clinical characteristics of severely or critically ill patients with COVID-19 and compare the clinical characteristics of the patients who received IRS with those of the patients who received NIRS. 


\section{MATERIALS AND METHODS}

\subsection{Study Design and Participants}

The patients were enrolled in this retrospective, single center study from January 8 to March 12, 2020, in the Intensive Care Unit (ICU) of Zhongnan Hospital of Wuhan University, a COVID-19-designated hospital in Wuhan, China. All patients with COVID-19 enrolled in this study were diagnosed according to the WHO interim guidance by detection of SARS-CoV-2 RNA or the typical CT scan hallmarks. Laboratory confirmation of COVID-19 was based on the positive detection of SARS-CoV-2 RNA, and the typical CT scan hallmark for COVID-19 was bilateral distribution of patchy shadows and ground glass opacity. Severe illness was defined according to any of the following items: (1) respiratory distress, respiratory rate $(\mathrm{RR}) \geq 30$ times/ $\min$; (2) in the resting state, oxygen saturation $\leq 93 \%$; or (3) arterial partial pressure of oxygen $\left(\mathrm{PaO}_{2}\right) /$ fraction of inspiration oxygen $\left(\mathrm{FiO}_{2}\right) \leq 300 \mathrm{mmHg}$ $(1 \mathrm{mmHg}=0.133 \mathrm{kPa})$. Critically ill patients met one of the following conditions: (1) respiratory failure needing mechanical ventilation; (2) shock; or (3) other organ failure needing ICU monitoring and treatment ${ }^{[7]}$.

\subsection{Data Collection}

The research team obtained epidemiological, clinical symptom, clinical sign, laboratory, treatment and outcome data from electronic medical records with pre-prepared data collection forms. Information recorded included demographic data, medical history, exposure history, underlying comorbidities, symptoms, signs, Acute Physiology and Chronic Health Evaluation II (APACHE II), Sequential Organ Failure Assessment (SOFA), laboratory findings, chest computed tomographic (CT) scans, and treatment measures (i.e., antiviral therapy, corticosteroid therapy, and respiratory support). The day when the symptoms were noticed was defined as the date of disease onset. APACHE II, SOFA and laboratory findings were collected at admission into ICU. All the data were checked by a trained team of physicians.

\subsection{Statistical Analysis}

Categorical variables were described as frequency rates and percentages, and continuous variables were described using mean, median, and interquartile range (IQR) values. Means for continuous variables were compared using independent group $t$ tests when the data were normally distributed; otherwise, the MannWhitney test was used. Data (nonnormal distribution) from repeated measures were compared using the generalized linear mixed model. Proportions for categorical variables were compared using the $\chi^{2}$ test, although Fisher's exact test was used when the data were limited. All statistical analyses were performed using SPSS (Statistical Package for the Social Sciences) version 26.0 software (SPSS Inc., USA). For unadjusted comparisons, a 2 -sided $\alpha$ of less than 0.05 was considered statistically significant. The analyses were not adjusted for multiple comparisons, and given the potential for type I error, the findings should be interpreted as exploratory and descriptive.

\section{RESULTS}

\subsection{Presenting Characteristics}

As of March 12, 2020, a total of 55 severely or critically ill patients were confirmed as suffering COVID-19 due to infection by SARS-CoV-2 in the ICU of Zhongnan Hospital of Wuhan University. All the patients were followed up as of March 12, 2020 when the patients were transferred to ICU of Wuhan Leishenshan Hospital, with the median followup of 26 days (IQR 16-35). The patients received different methods of respiratory support according to their breathing, including high-flow nasal cannula oxygen therapy (HFNCOT), non-invasive mechanical ventilation (NIMV), invasive mechanical ventilation (IMV) (table 1). According to whether the treatment was invasive, we divided these respiratory support modes into IRS and NIRS. The characteristics of patients who received IRS and NIRS are compared and shown in table 2.

Of the 55 patients, $5(9.1 \%)$ hospitalized patients who were already hospitalized for other reasons were presumed to have been infected in the hospital, and 4 $(7.3 \%)$ health care workers were infected in their work. Forty-six (83.6\%) COVID-19 patients were infected outside the hospital by contact with COVID-19 family members before isolation or during social activities. Only one patient had a exposure history of Huanan Seafood Wholesale Market (table 2).

The median age was 63.0 years (IQR, 53.0, 74.0; range, 22-92 years), and $34(61.8 \%)$ were men. The median duration from first symptoms to hospital

Table 1 The respiratory support of COVID-19 patients in ICU as of Mar. 12, 2020

\begin{tabular}{lcccccc}
\hline Characteristics & Total $(n=55)$ & HFNOT $(n=12)$ & NIMV $(n=15)$ & IMV $(n=19)$ & ECMO $(n=9)$ & $P$ value \\
\hline Age, median (IQR) & $63.0(54.0,74.0)$ & $61.5(48.0,76.8)$ & $64.0(56.0,74.0)$ & $65.0(61.0,81.0)$ & $50.0(31.0,65.5)$ & 0.010 \\
Men, $n(\%)$ & $34(61.8)$ & $7(58.3)$ & $8(53.3)$ & $15(78.9)$ & $4(44.4)$ & 0.590 \\
Women, $n(\%)$ & $21(38.2)$ & $5(41.7)$ & $7(46.7)$ & $4(21.1)$ & $5(56.6)$ & 0.590 \\
Death, $n(\%)$ & $16(29.1)$ & $1(8.3)$ & $1(5.7)$ & $10(52.6)$ & $4(44.4)$ & 0.005 \\
Discharge, $n(\%)$ & $33(60.0)$ & $11(91.7)$ & $13(86.7)$ & $6(31.6)$ & $3(33.3)$ & $<0.001$ \\
\hline
\end{tabular}

HFNCOT: high-flow nasal cannula oxygen therapy; NIMV: non-invasive mechanical ventilation; IMV: invasive mechanical ventilation; ECMO: extracorporeal membrane oxygenation 
Table 2 Baseline characteristics of COVID-19 patients in ICU

\begin{tabular}{|c|c|c|c|c|}
\hline Characteristics & Total $(n=55)$ & NIRS $(n=27)$ & $\operatorname{IRS}(n=28)$ & $P$ \\
\hline Age, median (IQR) & $63.0(54.0,74.0)$ & $64.0(54.0,74.0)$ & $62.5(52.5,71.3)$ & 0.783 \\
\hline Men, $n(\%)$ & $34(61.8)$ & $15(55.6)$ & $19(67.9)$ & 0.357 \\
\hline Huanan Seafood Wholesale Market exposure & $1(1.8)$ & $0(0.0)$ & $1(3.6)$ & 0.331 \\
\hline Population of infection & & & & 0.860 \\
\hline Hospitalized patients & $5(9.1)$ & $2(7.4)$ & $3(10.7)$ & \\
\hline Medical staff & $4(7.3)$ & $2(7.4)$ & $2(7.1)$ & \\
\hline Prehospital & $46(83.6)$ & $23(85.2)$ & $23(82.1)$ & \\
\hline \multicolumn{5}{|l|}{ Comorbidities } \\
\hline Hypertension & $25(45.5)$ & $12(44.4)$ & $13(46.4)$ & 0.885 \\
\hline Coronary artery disease & $11(20.0)$ & $4(14.8)$ & $7(25.0)$ & 0.354 \\
\hline Diabetes & $7(12.7)$ & $2(7.4)$ & $5(17.9)$ & 0.253 \\
\hline Malignancy & $1(1.8)$ & $1(3.7)$ & $0(0.0)$ & 0.313 \\
\hline Cerebrovascular disease & $5(9.1)$ & $0(0.0)$ & $5(17.9)$ & 0.021 \\
\hline COPD & $5(9.1)$ & $4(14.8)$ & $1(3.6)$ & 0.153 \\
\hline CKD & $2(3.6)$ & $1(3.7)$ & $1(3.6)$ & 0.980 \\
\hline Chronic liver disease & $2(3.6)$ & $2(7.4)$ & $0(0.0)$ & 0.148 \\
\hline \multicolumn{5}{|l|}{ Onset of symptom to, median (IQR), days } \\
\hline Hospital admission & $8.0(5.0,12.0)$ & $10.0(6.0,14.0)$ & $5.0(3.0,10.0)$ & 0.036 \\
\hline ICU admission & $10.0(7.0,15.0)$ & $11.0(8.0,15.0)$ & $7.5(4.3,14.8)$ & 0.191 \\
\hline HR, median (IQR), beats/min & $82.0(78.0,96.0)$ & $81.0(70.0,93.0)$ & $85.0(78.0,101.0)$ & 0.203 \\
\hline RR, median (IQR), & $22.0(20.0,25.0)$ & $22.0(20.0,24.0)$ & $23.0(21.0,25.8)$ & 0.153 \\
\hline MAP, median (IQR), mmHg & $92.0(82,100.0)$ & $88.0(80.0,97.0)$ & $95.3(82.5,100.0)$ & 0.200 \\
\hline
\end{tabular}

HR: heart rate; RR: respiratory rate; MAP: mean artery pressure; COPD: chronic obstructive pulmonary diseases; CKD: chronic kidney disease; NIRS: non-invasive respiratory support; IRS: invasive respiratory support

admission and ICU admission was 8 days (IQR, 5-12) and 10 days (IQR, 7-15), respectively (table 2). Of the 55 patients, $32(58.2 \%)$ had one or more coexisting medical conditions. Hypertension (25, 45.5\%), diabetes $(7,12.7 \%)$, and coronary artery disease $(11,20.0 \%)$ were the most common coexisting conditions. On admission, most patients had fever at the onset of illness (table 3). Other symptoms included fatigue, dry cough, dyspnea, expectoration, pharyngalgia and abdominal pain.

On admission, IRS-treated patients in the ICU had less lymphocyte counts than those who received NIRS. In addition, there were no significant differences between the two groups in their laboratory results, APACHE II and SOFA. Moreover, 39 (70.9\%) patients had a procalcitonin level above the normal range, including 15 (53.6\%) in the NIRS group and 24
Table 3 Initial symptoms of COVID-19 patients in ICU

\begin{tabular}{lcccc}
\hline Onset symptoms & $\begin{array}{c}\text { Total } \\
(n=55)\end{array}$ & $\begin{array}{c}\text { NIRS } \\
(n=27)\end{array}$ & $\begin{array}{c}\text { IRS } \\
(n=28)\end{array}$ & $P$ \\
\hline Fever $(n, \%)$ & $30(54.5)$ & $12(44.4)$ & $18(64.3)$ & 0.145 \\
Fatigue $(n, \%)$ & $5(9.1)$ & $4(14.8)$ & $1(3.6)$ & 0.153 \\
Dry cough $(n, \%)$ & $4(7.3)$ & $1(3.7)$ & $3(10.7)$ & 0.326 \\
Myalgia $(n, \%)$ & $3(5.5)$ & $3(11.1)$ & $0(0.0)$ & 0.072 \\
Dyspnea $(n, \%)$ & $3(5.5)$ & $2(7.4)$ & $1(3.6)$ & 0.540 \\
Expectoration $(n, \%)$ & $5(9.1)$ & $3(11.1)$ & $2(7.1)$ & 0.617 \\
Pharyngalgia $(n, \%)$ & $1(1.8)$ & $0(0.0)$ & $1(3.6)$ & 0.331 \\
Dizziness $(n, \%)$ & $3(5.5)$ & $1(3.7)$ & $2(7.1)$ & 0.583 \\
Abdominal pain $(n, \%)$ & $1(1.8)$ & $1(3.7)$ & $0(0.0)$ & 0.313 \\
\hline
\end{tabular}

$(88.9 \%)$ in the IRS group (table 4). The chest X-ray and CT findings in patients are shown in fig. 1. The typical findings of chest $\mathrm{CT}$ images of patients with COVID-19 were ground glass opacity in both lungs or single lung.
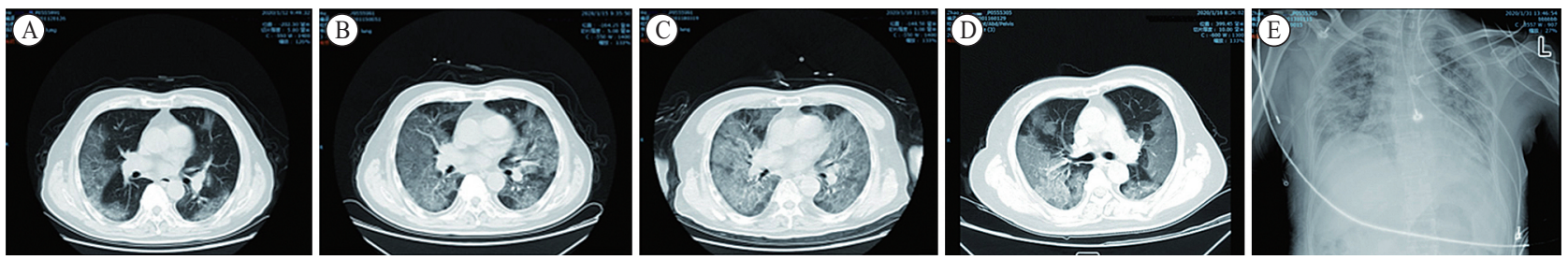

Fig. 1 Chest CT findings Images of a 72-years-old man. A: ground glass opacity in both lungs on Jan. 12; B and C: worsen 3 days (B) and 6 days (C) later. It was difficult to correct type II respiratory failure after invasive mechanical ventilation. VV-ECMO was performed on Jan. 18, withdrawn on Jan. 24 after conditions were improved. Tracheal intubation was pulled out on Jan. 27, and the patient was transferred out of ICU successfully. Images of a 65-year old women. D: chest CT scan showing ground glass opacity in both lungs; E: half a month later, chest X-ray showing the brightness of both lungs was decreased and multiple patchy shadows were observed. NIMV could ease her dyspnea and she discharged. 
Table 4 Laboratory results of COVID-19 patients in ICU

\begin{tabular}{|c|c|c|c|c|}
\hline Characteristics & Total $(n=55)$ & NIRS $(n=29)$ & $\operatorname{IRS}(n=26)$ & $P$ \\
\hline White blood cell count, $\times 10^{9} / \mathrm{L}$ & $9.7 \pm 6.0$ & $8.4 \pm 4.0$ & $10.0 \pm 6.3$ & 0.241 \\
\hline $\mathrm{HGB}, \mathrm{g} / \mathrm{L}$ & $120.0 \pm 284.9$ & $120.3 \pm 22.3$ & $121.2 \pm 23.7$ & 0.886 \\
\hline Lymphocyte count, $\times 10^{9} / \mathrm{L}$ & $0.6 \pm 0.4$ & $0.7 \pm 0.4$ & $0.5 \pm 0.3$ & 0.002 \\
\hline Platelet count, $\times 10^{9} / \mathrm{L}$ & $166.3 \pm 60.5$ & $184.9 \pm 66.8$ & $152.7 \pm 63.7$ & 0.075 \\
\hline Prothrombin time, s & $13.9 \pm 2.2$ & $14.1 \pm 2.2$ & $14.0 \pm 2.6$ & 0.948 \\
\hline FIB-C, mg/dL & $450.0 \pm 83.7$ & $479.6 \pm 165.7$ & $448.0 \pm 113.8$ & 0.665 \\
\hline D-dimer, mg/L & $3323.2 \pm 830.5$ & $2782.7 \pm 528.1$ & $5530.9 \pm 805.1$ & 0.228 \\
\hline Creatine kinase, $\mathrm{U} / \mathrm{L}$ & $364.2 \pm 81.0$ & $162.0 \pm 67.7$ & $461.7 \pm 118.1$ & 0.141 \\
\hline Creatine kinase-MB, U/L & $32.5 \pm 7.4$ & $22.0 \pm 6.6$ & $32.7 \pm 10.2$ & 0.130 \\
\hline Lactate dehydrogenase, $\mathrm{U} / \mathrm{L}$ & $499.7 \pm 204.1$ & $469.6 \pm 181.6$ & $502.8 \pm 208.5$ & 0.570 \\
\hline Alanine aminotransferase, $\mathrm{U} / \mathrm{L}$ & $47.1 \pm 19.5$ & $48.1 \pm 19.9$ & $47.1 \pm 12.4$ & 0.917 \\
\hline Aspartate aminotransferase, U/L & $73.9 \pm 12.4$ & $58.9 \pm 16.5$ & $68.1 \pm 12.6$ & 0.509 \\
\hline Total bilirubin, $\mathrm{mmol} / \mathrm{L}$ & $12.9 \pm 6.9$ & $14.6 \pm 6.4$ & $13.7 \pm 6.0$ & 0.678 \\
\hline Blood urea nitrogen, $\mathrm{mmol} / \mathrm{L}$ & $6.6 \pm 3.3$ & $6.5 \pm 2.8$ & $7.7 \pm 3.7$ & 0.344 \\
\hline Creatinine, $\mu \mathrm{mol} / \mathrm{L}$ & $95.9 \pm 26.2$ & $93.9 \pm 29.3$ & $87.9 \pm 38.1$ & 0.825 \\
\hline Hypersensitive troponin $\mathrm{I}, \mathrm{pg} / \mathrm{mL}$ & $102.7 \pm 45.1$ & $28.0 \pm 17.4$ & $189.8 \pm 45.4$ & 0.092 \\
\hline Procalcitonin, $\mathrm{ng} / \mathrm{mL}$ & $0.2(0.1,0.8)$ & $0.1(0.1,0.4)$ & $0.6(0.1,4.3)$ & 0.106 \\
\hline Procalcitonin, $\mathrm{ng} / \mathrm{mL} \geq 0.05, n(\%)$ & $39(70.9)$ & $15(53.6)$ & $24(88.9)$ & 0.003 \\
\hline \multicolumn{5}{|l|}{ Baseline arterial blood gases } \\
\hline $\mathrm{PaCO}_{2}, \mathrm{mmHg}$ & $35.5 \pm 6.8$ & $34.8 \pm 6.0$ & $36.2 \pm 7.7$ & 0.464 \\
\hline $\mathrm{PaO}_{2}, \mathrm{mmHg}$ & $75.3 \pm 28.2$ & $71.0 \pm 23.7$ & $79.5 \pm 29.2$ & 0.416 \\
\hline $\mathrm{PaO}_{2} / \mathrm{FiO}_{2}, \mathrm{mmHg}$ & $174.4 \pm 56.3$ & $193.0 \pm 50.7$ & $156.4 \pm 51.8$ & 0.204 \\
\hline APACHE II & $18.7 \pm 6.0$ & $18.2 \pm 6.5$ & $19.3 \pm 5.6$ & 0.629 \\
\hline SOFA & $7.5 \pm 3.3$ & $7.9 \pm 3.8$ & $7.2 \pm 2.8$ & 0.572 \\
\hline
\end{tabular}

HBG: hemoglobin; FIB-C: fibrinogen-c; $\mathrm{PaCO}_{2}$ : partial pressure of carbon dioxide in artery; $\mathrm{PaO}_{2}$ :arterial partial pressure of oxygen; $\mathrm{PaO}_{2} / \mathrm{FiO}_{2}$ : the rate of arterial partial pressure of oxygen and fraction of inspiration oxygen; APACHE II: acute physiology and chronic health evaluation II; SOFA: sequential organ failure assessment

All 55 ICU patients received antiviral therapy, including oseltamivir $(48,87.3 \%)$, arbidol $(4,7.2 \%)$ or kaletra $(5,9.1 \%)$ (table 5). In addition, $45(81.8 \%)$ patients received glucocorticoid therapy, including 21 (75\%) patients in the NIRS group and $24(88.9 \%)$ in the IRS group.

All the patients were followed up as of March 12 with the median follow-up of 26 days (IQR 16-35). Of the 55 severely or critically ill ICU patients, 39 $(70.9 \%)$ were still alive as of March 12, $6(10.9 \%)$ patients transferred to isolation wards, and $33(60.0 \%)$ discharged successfully. Sixteen (29.1\%) patients died during the follow-up, and the patients in the IRS group had a higher mortality rate than those in the NIRS group ( $\mathrm{HR}=7.90,95 \%$ CI $1.98-14.50$, $P<0.05$ ) (table 5 and fig. 2). Nine patients received venovenous extracorporeal membrane oxygenation (VV-ECMO) for respiratory support after active treatment but failed due to severe respiratory failure. Five patients had successfully discontinued ECMO, 3 patients discharged, and 2 were put in quarantine. The other four patients died. One case of ECMO failure was an old man and he was complicated with secondary severe infection and developed into septic shock and multiple organ failure after ECMO support. Another one was a 31-year-old pregnant woman that discontinued treatment for financial reasons because the government had not implemented the policy of a

Table 5 Treatment and prognosis of COVID-19 patients in ICU

\begin{tabular}{|c|c|c|c|c|}
\hline Characteristics & Total $(n=55)$ & NIRS $(n=29)$ & $\operatorname{IRS}(n=26)$ & $P$ \\
\hline \multicolumn{5}{|l|}{ Treatment } \\
\hline Antiviral therapy & $55(100.0)$ & $27(100.0)$ & $28(100.0)$ & - \\
\hline Oseltamivir, $n(\%)$ & $48(87.3)$ & $25(92.6)$ & $23(82.2)$ & \\
\hline Arbidol, $n(\%)$ & $4(16.4)$ & $2(7.4)$ & $2(7.1)$ & \\
\hline Kaletra, $n(\%)$ & $5(9.1)$ & $3(11.1)$ & $2(7.1)$ & \\
\hline Glucocorticoid therapy, $n(\%)$ & $45(81.8)$ & $20(74.1)$ & $25(89.3)$ & 0.149 \\
\hline Antibiotic therapy, $n(\%)$ & $55(100.0)$ & $27(100.0)$ & $28(100.0)$ & - \\
\hline \multicolumn{5}{|l|}{ Prognosis as of March 12} \\
\hline Death $(n, \%)$ & $16(29.1)$ & $2(7.4)$ & $14(50.0)$ & $<0.001$ \\
\hline Isolation wards $(n, \%)$ & $6(10.9)$ & $1(3.7)$ & $5^{*}(17.9)$ & 0.001 \\
\hline Discharge $(n, \%)$ & $33(60.0)$ & $24(88.9)$ & $9(32.1)$ & $<0.001$ \\
\hline
\end{tabular}

${ }^{*}$ Two out of five patients were transferred to ICU of Wuhan Leishenshan Hospital, and rest two were put in quarentine. 


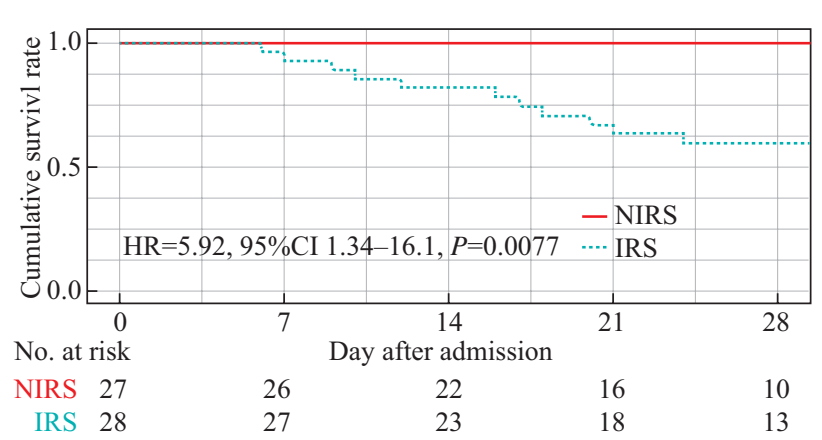

Fig. 2 Kaplan-Meier curves of the patients stratified by noninvasive respiratory support (NIRS) and invasive respiratory support (IRS)

minimum cost guarantee at that time. The other two experienced cardiopulmonary resuscitation just before or after performing ECMO with poor neurological function and died of multiple organ failure.

\section{DISCUSSION}

Here we present a descriptive study on the difference of the clinical and epidemiological characteristics of the COVID-19 between the two groups with different respiratory support method. Once the patients breathe so difficultly and need IRS, the prognosis was relatively poor. ECMO can be taken into consideration if the patient's condition permits. So we should, on the one hand, try our best to reverse the severe condition and avoid IRS, and on the other hand, grasp the timing of intervention of IRS, in some time when early intervention is needed.

COVID-19, with the main pathogen of SARS$\mathrm{CoV}-2$, is capable of efficient transmission among humans ${ }^{[8,9]}$. The virus can cause acute respiratory infection symptoms, and ARDS and acute respiratory failure also occur frequently in some patients. After infection, most patients present with fever, dry cough, dyspnea, and bilateral ground glass opacities on chest CT scans. However, few patients with 2019-nCoV infection have prominent upper respiratory tract signs and symptoms (e.g., rhinorrhea, sneezing, or sore throat), indicating that the target cells might be located in the lower airway. The highly infectious COVID-19 can lead to fatal complication especially COVID-19-related ARDS. Thus, fully understanding the characteristics of COVID-19-related ARDS is important to early identification and precise treatment.

Recent evidence indicated that patients infected with SARS-CoV-2 had high amounts of serum IL1B, IFN $\gamma$, IP10, and MCP1, probably leading to activated $\mathrm{T}$ helper-1 (Th1) cell responses ${ }^{[9]}$. Therefore, lung function injury after infection of SARS-CoV-2 may be associated with cytokine storms and low immune function. Xu et al have explored the pathological characteristics of a 50-year-old COVID-19 patient by minimally invasive autopsy. In their study, they found that interstitial mononuclear inflammatory infiltrates, dominated by lymphocytes, are observed in both lungs ${ }^{[10]}$. This study also reveals evident desquamation of pneumocytes and formation of hyaline membrane on the right lung, and the left lung tissue appears as pulmonary oedema with hyaline membrane formation, which is suggestive of $\mathrm{ARDS}^{[10]}$. In our study, the absolute value of lymphocytes in most patients was reduced. Mostly, the level of lymphocytes in patients receiving IRS was lower than that in those receiving NIRS, and the level of lymphocytes was elevated when the condition improved. This result suggests that SARS-CoV-2 might act mainly on lymphocytes, especially $\mathrm{T}$ lymphocytes, which causes lung injury and ARDS, eventually followed by multiple organ failure.

ARDS is a life-threatening form of respiratory failure characterized by inflammatory pulmonary edema resulting in severe hypoxemia ${ }^{[11]}$, and the treatments of ARDS include mechanical ventilation, corticosteroids and so on. Corticosteroids were used frequently for the treatment of patients with ARDS for possible benefit by reducing inflammatory-induced lung injury. But the effects of administration of corticosteroids in COVID19-related ARDS patients were uncertain. The results of a recent meta-analysis showed that corticosteroid treatment did not bring clinical benefit to the mortality rate but rather delayed viral clearance and brought about a series of relevant side effects ${ }^{[12]}$. Mechanical ventilation is the main supportive treatment for critically ill patients and supportive care with mechanical ventilation remains the cornerstone of ARDS management ${ }^{[13]}$. Mechanical ventilation itself can cause and potentiate lung injury, namely, ventilator-induced lung injury ${ }^{[14]}$. A lung-protective ventilation strategy and early medical management for patients with severe ARDS are recommended ${ }^{[15]}$ and recommendations for ECMO were in line with other causes of severe ARDS refractory to ARDSnet therapy ${ }^{[16]}$. The newly published guidelines in JAMA suggest that invasive mechanical ventilation can be considered, and the principle of "lung-protective ventilation should be performed when severe ARDS was diagnosed"[17]. In our study, NIMV can be safe in COVID-19-related ARDS patients, even in some moderate-severe patients. But many patients progressed rapidly from NIRS to IRS. Even though the recommended lung-protective ventilation strategy was performed, the condition deteriorated so quickly that only ECMO could sustain life by sparing some time for lung repair. A study from our center reported that 21 patients with mechanical ventilation received ECMO support and 12 patients died and 9 survived by April 7, 2020 ${ }^{[18]}$. ECMO might be an effective salvage treatment for patients with SARS-CoV-2 pneumonia associated with severe ARDS. Moreover, the damaged 
lungs tend to worsen under high mechanical ventilation parameters; therefore, it could be expeditious to start ECMO as early as possible, especially after lung protective ventilation (tidal volume $6 \mathrm{~mL} / \mathrm{kg}$, PEEP $\geq 10 \mathrm{cmH}_{2} \mathrm{O}$ ) was adopted and combined with lung recruitment maneuver, prone position ventilation, and high-frequency oscillation ventilation, patients are still under the condition of pure oxygen inhalation.

This study has several limitations. It would be beneficial to include additional patients from other hospitals in Wuhan, in other cities in China, and even in other countries to obtain a more comprehensive understanding of the respiratory support strategy of patients suffering from COVID-19-related ARDS. However, the datain this study permit an early assessment of the epidemiological and clinical characteristics of different respiratory support method in severely or critically ill ICU patients with COVID-19. Further researches on the effects of the timing of invasive mechanical ventilation on prognosis are necessary to help clinicians to make correct strategies in the face of challenge of ARDS. However, additional efforts should be also made to obtain a full understanding of SARSCoV-2 and COVID-19, particularly the preparation of virus vaccines and researches on the development of effective antiviral drugs, in future studies.

The conclusions from our single-center case series of severely or critically ill ICU patients with COVID-19 in Wuhan, China, presumed that severely or critically ill ICU patients with COVID-19 have an increased likelihood to develop severe ARDS or severe acute respiratory failure, with a mortality of $50 \%$ for those requiring IRS when NIRS cannot ease their symptoms and respiratory failure cannot be rectified. ECMO can be taken into consideration if the condition permits. However, the time of intervention needs to be further discussed.

\section{Conflict of Interest Statement}

All authors declare that there is no conflict of interest and financial interest.

\section{REFERENCES}

1 Zhu N, Zhang D, Wang W, et al. A Novel Coronavirus from Patients with Pneumonia in China, 2019. N Engl J Med, 2020,382(8):727-733

$2 \mathrm{Lu}$ H, Stratton CW, Tang YW. Outbreak of pneumonia of unknown etiology in Wuhan, China: The mystery and the miracle. J Med Virol, 2020,92(4):401-402

3 Hui DS, I AE, Madani TA, et al. The continuing 2019$\mathrm{nCoV}$ epidemic threat of novel coronaviruses to global health - The latest 2019 novel coronavirus outbreak in Wuhan, China. Int J Infect Dis, 2020,91:264-266

4 Phan LT, Nguyen TV, Luong QC, et al. Importation and
Human-to-Human Transmission of a Novel Coronavirus in Vietnam. N Engl J Med, 2020,382(9):872-874

5 Epidemiology Working Group for NCIP Epidemic Response, Chinese Center for Disease Control and Prevention. The epidemiological characteristics of an outbreak of 2019 novel coronavirus diseases (COVID-19) in China. Zhonghua Liu Xing Bing Xue Za Zhi (Chinese), 2020,41(2):145-151

6 Wang $\mathrm{D}, \mathrm{Hu} \mathrm{B}, \mathrm{Hu} \mathrm{C}$, et al. Clinical Characteristics of 138 Hospitalized Patients With 2019 Novel Coronavirus-Infected Pneumonia in Wuhan, China. JAMA, 2020,323(11):1061-1069

7 Song F, Shi N, Shan F, et al. Emerging 2019 Novel Coronavirus (2019-nCoV) Pneumonia. Radiology, 2020, 295(1):210-217

8 Chan JF, Yuan S, Kok KH, et al. A familial cluster of pneumonia associated with the 2019 novel coronavirus indicating person-to-person transmission: a study of a family cluster. Lancet, 2020,395(10223):514-523

9 Huang C, Wang Y, Li X, et al. Clinical features of patients infected with 2019 novel coronavirus in Wuhan, China. Lancet, 2020,395(10223):497-506

$10 \mathrm{Xu} \mathrm{Z}$, Shi L, Wang Y, et al. Pathological findings of COVID-19 associated with acute respiratory distress syndrome. Lancet Respir Med, 2020,8:420-422

11 Wang C, Horby PW, Hayden FG, et al. A novel coronavirus outbreak of global health concern. Lancet, 2020,395(10223):470-473

12 Ware LB, Matthay MA. The acute respiratory distress syndrome. N Engl J Med, 2000,342(18):1334-1349

13 Russell CD, Millar JE, Baillie JK. Clinical evidence does not support corticosteroid treatment for 2019nCoV lung injury. Lancet, 2020,395(10223):473-475

14 Fan E, Needham DM, Stewart TE. Ventilatory management of acute lung injury and acute respiratory distress syndrome. JAMA, 2005,294(22):2889-2896

15 Slutsky AS, Ranieri VM. Ventilator-induced lung injury. N Engl J Med, 2013,369(22):2126-2136

16 Brochard L, Slutsky A, Pesenti A. Mechanical Ventilation to Minimize Progression of Lung Injury in Acute Respiratory Failure. Am J Respir Crit Care Med, 2017,195(4):438-442

17 Brodie D, Slutsky AS, Combes A. Extracorporeal Life Support for Adults With Respiratory Failure and Related Indications: A Review. JAMA, 2019,322(6):557-568

18 Fichtner F, Moerer O, Weber-Carstens S, et al. Clinical Guideline for Treating Acute Respiratory Insufficiency with Invasive Ventilation and Extracorporeal Membrane Oxygenation: Evidence-Based Recommendations for Choosing Modes and Setting Parameters of Mechanical Ventilation. Respiration, 2019,98(4):357-372

19 Yang X, Cai S, Luo Y, et al. Extracorporeal Membrane Oxygenation for Coronavirus Disease 2019-Induced Acute Respiratory Distress Syndrome: A Multicenter Descriptive Study. Crit Care Med, 2020. doi: 10.1097/ CCM.0000000000004447. Online ahead of print (Received May 26, 2020; accepted July 10, 2020) 